\title{
Contribution au développement de nouvelles techniques pour la détection des rayonnements cosmiques
}

\author{
A. MISHEV ${ }^{1,3}$, I. ANGELOV ${ }^{2}$, S.C. MADROVIEV ${ }^{1}$, J. STAMENOV ${ }^{1}$, \\ R. GSCHWIND ${ }^{3}$, L. MAKOVICKA ${ }^{3}$
}

(Manuscrit reçu le 18 juin 2002, accepté le 31 janvier 2003)

RÉSUMÉ Les investigations du rayonnement cosmique font partie à la fois de la physique des particules élémentaires, de l'astrophysique, de l'environnement, de la radioprotection et des techniques instrumentales liées à toutes ces disciplines. L'évolution technique et scientifique actuelle met de plus en plus souvent I'homme et le matériel dans des conditions d'exposition radiative quasipermanente (espace énergétique - milieu médical - applications industrielles). Le développement des techniques de détection et la bonne connaissance des champs de rayonnement représentent en général une base essentielle pour une radioprotection efficace et préventive. Les études effectuées au cours de ce travail ont mis en évidence de nombreux phénomènes tels que les cascades électromagnétiques ou l'effet Tcherenkov. Les variations du rayonnement cosmique peuvent être évaluées avec un télescope muonique et des détecteurs Tcherenkov à base d'eau. De plus, la détection des particules les plus énergétiques du rayonnement cosmique peut être basée sur l'enregistrement des photons Tcherenkov créés dans l'atmosphère. Ainsi, ces nouvelles techniques jouent un rôle déterminant dans la recherche du spectre et la composition du rayonnement cosmique primaire. Ce travail a permis l'utilisation de différents codes Monte-Carlo pour simuler des cascades électromagnétiques et hadroniques dans différents milieux (EGS4 et CORSIKA 5.62). La partie « utilisateur » du code EGS4 a été complétée par l'effet Tcherenkov pour modéliser cet effet dans l'eau et simuler la réponse d'un détecteur concret. Les résultats obtenus ont permis d'estimer l'efficacité du télescope muonique développé à l'université de Blagoevgrad en Bulgarie et des études expérimentales liées à ce modèle ont servi à optimiser le fonctionnement du télescope muonique et donc à améliorer ses performances. Différentes caractéristiques des grandes gerbes de l'air, plus précisément la fonction de la distribution latérale du rayonnement Tcherenkov ont été obtenues par le code CORSIKA 5.62. Une approximation analytique a été trouvée. Une méthode basée à la fois sur la solution d'un problème inverse et sur l'enregistrement des photons Tcherenkov a été proposée pour déterminer la composition du rayonmement cosmique et pour estimer son spectre.

ABSTRACT Contribution to the development of new techniques for cosmic rays detection.

The researches about cosmic rays belong to the domain of elementary particle physics, environment, astrophysics, radiation protection and of the instrumental techniques connected to these scientific disciplines. Current technical and scientific

\footnotetext{
IRNEN, Bulgarian Academy of Sciences, Tsarigradsko chaussé 72, 1784 Sofia, Bulgarie.

2 Université Neofirt Rilski, Département de Physique, Blagoevgrad, Bulgarie.

3 CREST (UMR 6000 CNRS), Pôle universitaire, B.P. 71427, 25211 Montbéliard, France
} 
evolution more and more often puts the man and the material in the conditions of radiative exposure almost permanent (energy, space, medical environment, industrial applications). The development of detection techniques and the good knowledge of the fields of radiation represent a base of essential data for an effective radiation protection. Many phenomena such as electromagnetic showers, Cerenkov effect have been highlighted by the studies made about this paper. The development of this domain is rapidly expanding for some years. The investigations of the cosmic ray variations can be made using a muonic telescope with water Cerenkov detectors and also the detection of the most energy particles of the cosmic rays is based on atmospheric Cerenkov technique. The application of new techniques is important to resolve problems such the composition and the spectrum of the primary cosmic radiation. This work has used various Monte Carlo codes to simulate electromagnetic and hadronic showers in different media (EGS4 and CORSIKA 5.62). The user part of the EGS4 code was completed by the Tcherenkov effect which permits to simulate a concrete detector. The modelling of the Cerenkov effect in water (EGS4) allowed to estimate the efficiency of the muonic telescope developed in Blagoevgrad's University in Bulgaria. The experimental studies connected to this model are the foundation of a methodology to optimise the telescope. Using the CORSIKA 5.62 code the various characteristics of the extensive air showers, specially the lateral distribution function of Cerenkov light, were obtained. An analytical approximation of this distribution was obtained. A methodology based on the solution of an inverse problem and the Cerenkov photons registration were proposed to determine the mass composition of cosmic rays and the estimation of their spectra.

\section{Introduction}

Au fil des années, les études du rayonnement cosmique (variation de sa fluence et des doses reçues) ont été liées aux travaux de la radioprotection et de l'environnement. Actuellement, le développement de nouvelles techniques, destinées par exemple à l'évaluation de la transparence atmosphérique (présence des différents polluants, aérosols, etc.), prend de plus en plus d'ampleur. C'est dans ce contexte que s'inscrit ce travail. Il entre dans le cadre du programme de recherches de l'Académie des sciences de Bulgarie (Angelov et al., 1999) et des collaborations franco-bulgares (projet OM2, thèse de A. Mishev) entre l'Institut de la recherche et de l'énergie nucléaire de Sofia et le Centre de recherche sur les écoulements, les surfaces et les transferts de Montbéliard (Duverger et al., 2000 ; Mishev et al., 2001 a, 2001b ; Mishev, 2002). Il est consacré aux méthodes Monte Carlo et plus particulièrement à la modélisation de l'effet Tcherenkov dans différents milieux. L'objectif principal de cette étude a porté sur l'amélioration de l'appareillage, notamment sur le régime de fonctionnement d'un télescope muonique construit avec des détecteurs Tcherenkov à base d'eau, qui est installé à l'université de Blagoevgrad (Bulgarie). L'élaboration d'un modèle théorique des processus physiques concernés (simulation des cascades électromagnétiques dans différents milieux pour différentes géométries) avec le code EGS4 a permis d'estimer son efficacité de détection. L'effet Tcherenkov, absent jusqu'à présent dans la version originale de ce logiciel de recherche, a été introduit. La vérification 
des modèles a été effectuée, d'une part à l'aide des études expérimentales et d'autre part, grâce à la comparaison avec le code CORSIKA qui utilise une version d'EGS4 modifiée.

Depuis quelques années la détection des particules de plus en plus énergétiques du rayonnement cosmique est basée sur l'enregistrement des photons Tcherenkov dans l'atmosphère. L'amélioration de la statistique et l'application de nouvelles techniques y jouent un rôle important. Le développement d'une méthodologie basée uniquement sur la détection des rayons Tcherenkov semble être d'un grand intérêt, surtout pour des projets récents ou en préparation. L'exploitation du code CORSIKA (code mis en place au KFK Karlsruhe, incluant les résultats les plus récents obtenus sur les accélérateurs du CERN) pouvait permettre d'obtenir les données nécessaires à l'évolution des modèles Monte Carlo des grandes gerbes dans l'air.

La bonne connaissance des phénomènes radiatifs peut donc non seulement contribuer à une radioprotection préventive mais aussi à la surveillance de l'environnement, car l'effet Tcherenkov est étroitement lié à l'indice de réfraction de l'air. Il pourrait, par exemple, être exploité dans certaines conditions comme indicateur des pollutions atmosphériques.

\section{Télescope muonique}

Le rayonnement cosmique primaire représente un flux isotrope de particules de haute et de très haute énergie (dont environ $90 \%$ sont des protons). Pendant les interactions du rayonnement primaire avec l'atmosphère naît la partie secondaire. Les variations de la partie muonique sont liées aux changements de l'intensité du flux primaire du rayonnement cosmique liée à la composante solaire, ainsi qu'à ceux du champ magnétique terrestre et de l'activité du Soleil.

Le télescope est conçu pour mesurer les variations de la composante muonique du rayonnement cosmique. Il est placé au sous-sol du bâtiment de l'université de Blagoevgrad «Neofirt Rilski » en Bulgarie. Il est construit en deux modules identiques (deux plans parallèles) comportant chacun 9 détecteurs de Tcherenkov à base d'eau (Figs. la et lb) (Angelov et al., 1999).

Les détecteurs sont des cuves de verre avec une couche d'aluminium déposée sur la surface interne qui sert de réflecteur (à l'exception des entrées optiques du photomultiplicateur PM). On utilise comme radiateur de l'eau distillée pour éviter l'absorption des photons et ainsi diminuer la perte du signal utile. L'enregistrement des photons Tcherenkov s'effectue à l'aide des photomultiplicateurs de type 


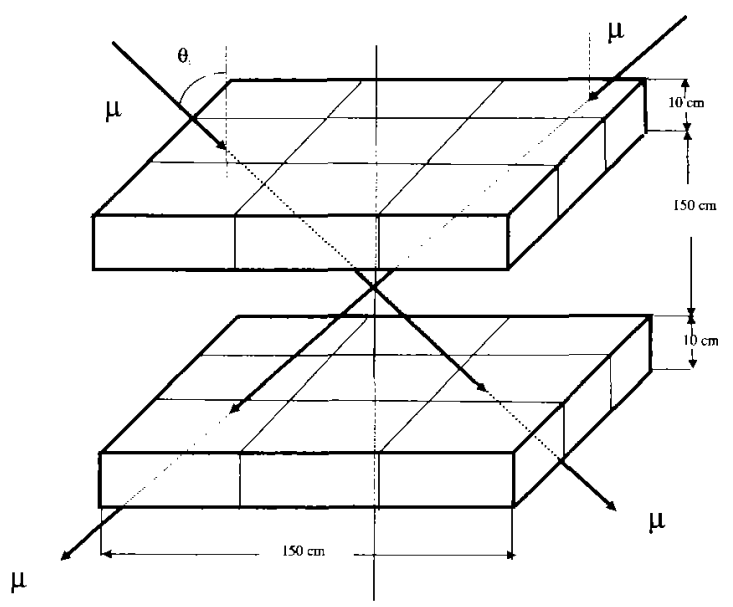

(a)

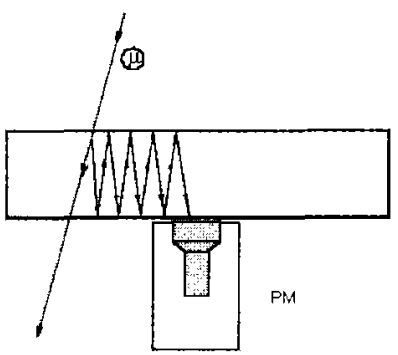

(b)

Figure 1 - (a) Schéma du télescope muonique. (b) Schéma d'une cuve du télescope avec le PM.

(a) Scheme of the muonic telescope. (b) Scheme of the telescope box with PM.

FEU-110 et FEU-139, ayant des caractéristiques identiques, situés au centre des cuves (un PM par cuve). Le diamètre des PM est de $65 \mathrm{~mm}$.

Les dimensions des cuves sont les suivantes : $X=49 \mathrm{~cm}, Y=49 \mathrm{~cm}$ et $Z=$ $12,5 \mathrm{~cm}$. La hauteur d'eau dans les cuves est fixée à $10 \mathrm{~cm}$ suite à des études expérimentales préalables et des critères pratiques liés à sa mise en place. Ce niveau permet bien d'exploiter le télescope pour enregistrer la partie muonique. Selon les estimations théoriques, son efficacité pour l'enregistrement des autres parties du rayonnement cosmique est faible (par exemple la détection des neutrinos). Bien que le télescope à muons ait un faible pouvoir de résolution, il donne des résultats comparables à ceux obtenus avec d'autres appareillages (Malamova et al., 2001).

L'ensemble des deux cuves représente un angle solide dans lequel le rayonnement cosmique secondaire est enregistré, essentiellement la composante muonique car les électrons sont absorbés dans les couches successives de béton. La distance entre les deux cuves est connue et à l'aide des schémas de coïncidence, on peut mesurer l'intensité de la partie muonique pour différents angles solides et pour différentes directions. 
Le télescope, situé au sous-sol de l'université, est protégé par 5 plaques de béton, qui selon les estimations théoriques sont en totalité équivalentes à un absorbeur de $430 \mathrm{~g} \mathrm{~cm}^{-2}$. Cela permet de rejeter les électrons secondaires du rayonnement cosmique. Pour éviter des événements parasites provoqués par les électrons delta produits essentiellement par les interactions dans la dernière plaque de béton, une couche supplémentaire de $5 \mathrm{~cm}$ de $\mathrm{Pb}$ a été placée entre les deux

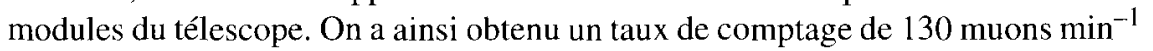
pour une paire de cuves.

De nombreux mesures additionnelles (Mishev et al., 2001a, 200lb ; Mishev, 2002) ont été effectuées pour choisir et optimiser les paramètres de fonctionnement. Une carte électronique assurant le fonctionnement automatique du télescope a été même développée à l'IRNEN de Sofia.

Comme une partie des paramètres physiques initiaux sont empiriques, il a semblé utile pour leur meilleure maîtrise de développer un modèle théorique permettant de simuler l'effet Tcherenkov.

\section{Association de l'effet Tcherenkov au code EGS4 et vérification expérimentale}

Le but principal de l'association de l'effet Tcherenkov au code EGS4 est d'obtenir l'estimation approximative de la réponse des détecteurs constituants le télescope muonique dans des conditions spécifiques (dimensions limitées, bande d'énergie limitée) afin de maîtriser sur le plan théorique la plupart des paramètres permettant un meilleur contrôle du fonctionnement de l'installation.

La plupart des codes de Monte Carlo développés pour la simulation des GGA (grandes gerbes dans l'air) ou des détecteurs utilisés pour l'enregistrement de la partie secondaire des rayonnements cosmiques sont généralement limités à un milieu - l'atmosphère (ex. code CORSIKA) (Heck et al., 1998).

EGS4 (Nelson et al., 1985) est un code Monte-Carlo bien connu et utilisable aussi pour la simulation des cascades électromagnétiques, le transport des électrons et des photons, leurs interactions dans différents milieux et dans une bande d'énergie de quelques keV à $1 \mathrm{TeV}$. Malgré ses performances, la version originale de ce code ne donnait jusqu'à présent ni la possibilité de simuler l'effet Tcherenkov, ni de suivre des particules telles que les muons.

Il est composé de deux sous-programmes, appelés par le programme utilisateur principal MAIN : ce sont HATCH et SHOWER. Le sous-programme HATCH permet de lire les données (sections efficaces) calculées par PEGS4 et le 
sous-programme SHOWER génère la simulation d'une histoire. SHOWER utilise à la fois la partie intrinsèque du code concernant les processus physiques d'interaction des électrons et des photons et la partie écrite par l'utilisateur. Cette dernière comprend HOWFAR, spécialiste de la géométrie, qui détermine la position de la particule courante et AUSGAB qui permet la mise en forme des résultats.

La mise en place de l'effet Tcherenkov dans le code EGS4 est basée sur le principe utilisé dans la version modifiée d'EGS4 du code CORSIKA mais sans la réduction du pas de transport.

Les changements ont été effectués directement dans AUSGAB (Mishev, 2002). Un sous-programme complémentaire TCHERE pour la production des photons Tcherenkov a été utilisé. Pour des raisons pratiques les premières simulations ont été faites pour un radiateur d'eau. Ainsi la modélisation de la réponse d'un compteur Tcherenkov à base d'eau en réflexion totale a été réalisée.

Le sous-programme TCHERE qui « produit » les photons Tcherenkov utilise les variables: IRL qui donne l'indice de la région d'intérêt, GAMMA le facteur de Lorenz, BETA $(\beta=v / c)$. INDEX représente l'indice de réfraction du milieu (modification de BETA) et TVSTEP la distance de transport pendant laquelle l'énergie de la particule chargée est constante, c'est-à-dire qu'elle est au-dessus du seuil de l'effet Tcherenkov. Les pertes en énergie dues à l'effet Tcherenkov ne sont pas prises en compte car elles sont négligeables par rapport aux pertes par ionisation.

La simulation de l'angle d'émission n'a pas été réalisée dans une première approche, car la simulation concerne un compteur en réflexion totale. Tous les photons Tcherenkov dans le radiateur sont donc enregistrés.

Les résultats des simulations effectuées ont été obtenus pour différentes géométries, correspondant à différentes cuves en réflexion totale, pour des électrons et des photons gamma dans une large bande énergétique. Ce sont surtout les électrons Compton qui donnent des photons Tcherenkov. Dans ce cas précis, pour les basses énergies, la probabilité de l'effet photoélectrique n'est pas très grande pour l'eau. Pour les hautes énergies ce sont surtout des particules chargées créées par la production des paires électrons-positrons qui produisent des photons Tcherenkov. Ainsi, un détecteur idéal qui enregistre tous les photons Tcherenkov produits dans la cuve a été simulé.

Quelques résultats de simulation d'un détecteur Tcherenkov à base d'eau en réflexion totale en utilisant ce simple modèle sont présentés sur les figures 2 


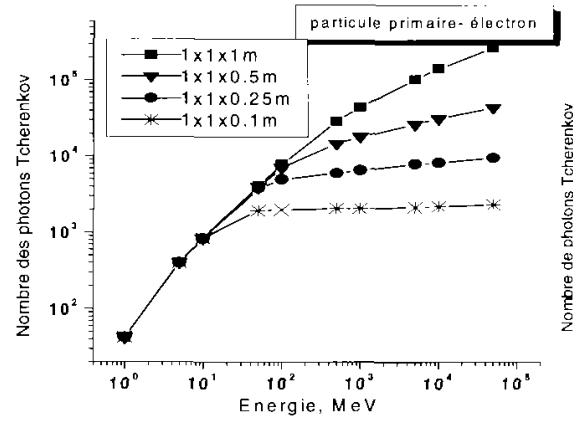

(a)

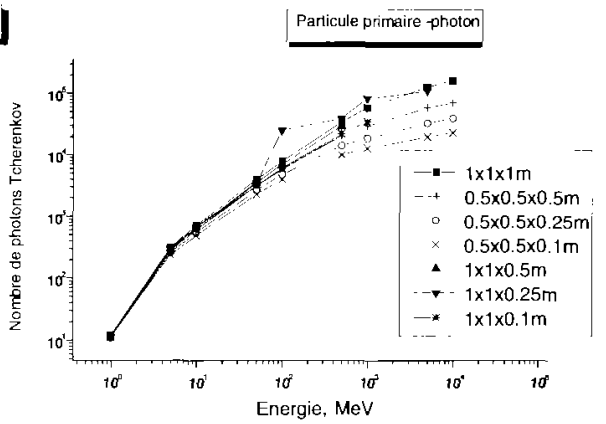

(b)

Figure 2 - (a) Simulations de la réponse aux électrons primaires d'un détecteur Tcherenkov en réflexion totale effectuées pour différentes profondeurs d'eau et avec I $0^{4}$ histoires par énergie. (b) Simulations de la réponse aux gamma primaires d'un détecteur Tcherenkov en réflexion totale effectuées pour différentes profondeurs d'eau et avec $10^{4}$ histoires par énergie.

(a) Simulations of the response to the primary electrons of a Cerenkov detector in total reflection made for various depths of water and with $10^{4}$ histories by energy. (b) Simulations of the response to the primary gamma of a Cerenkov detector in total reflection made for various depths of water and with $10^{4}$ histories by energy.

(Figs. 2a et 2b). La particule primaire incidente se déplace perpendiculairement au socle de la cuve.

La bande d'énergie varie entre $1 \mathrm{MeV}$ et $100 \mathrm{GeV}$. Il existe une dépendance en fonction de l'énergie et de la géométrie. Une saturation de photons Tcherenkov a été observée. Le nombre de particules chargées n'augmente plus ; toutes les particules secondaires produisent presque le même nombre de photons Tcherenkov car elles sont toutes ultra relativistes. Donc au-dessus d'une certaine énergie, le nombre des photons Tcherenkov émis par une particule est presque constant et le nombre de particules secondaires qui peuvent produire des photons Tcherenkov est également quasi constant. On constate qu'il n'y a presque pas de dépendance du nombre des photons Tcherenkov en fonction de la largeur de la cuve.

Une vérification expérimentale directe a été effectuée. Cette vérification expérimentale a été basée essentiellement sur le fonctionnement du télescope muonique construit à l'université de Blagoevgrad. Pour pouvoir vérifier le modèle simple introduit dans EGS4 (l'effet Tcherenkov), on a utilisé une source gamma isotopique ${ }^{60} \mathrm{Co}$ présentant une énergie moyenne au-dessus du seuil de l'effet Tcherenkov dans l'eau et l'appareillage existant du télescope muonique. 
En principe dans notre cas il est impossible de distinguer un signal donné par un muon du rayonnement cosmique d'un autre signal donné par le gamma du ${ }^{60} \mathrm{Co}$. La différence se situe dans le nombre des photons Tcherenkov émis dans la cuve. Cependant l'étalonnage du télescope n'avait pas été effectué, il ne travaille qu'en régime de compteur. Une protection électronique a été élaborée pour limiter au minimum les évènements créés par des muons considérés dans ce cas comme bruit de fond. En effet, cette protection était basée sur un schéma d'anticoïncidences entre le télescope muonique et le scintillateur. Le schéma suivant a été utilisé : un événement a été enregistré dans le cas d'anticoïncidence entre le télescope et le module de mesure (scintillateur en coïncidence avec la cuve inférieure) présenté sur la figure 3a. En effet, dans les études préliminaires une cuve moins large mais plus profonde (Fig. 3b) a été utilisée (Mishev et al., 2001a, 2001b ; Mishev, 2002). Cela nous a permis d'obtenir des nombres différents des photons Tcherenkov produits dans la cuve (recherche de la liaison entre le courant de sortie et le nombre des photons enregistrés par le PM). La réponse expérimentale de la cuve (taux de comptage en fonction du seuil du PM pour 10,20 et $30 \mathrm{~cm}$ de l'eau) est montrée sur la figure 3c (Mishev et al., 2001a, 2001b). Chaque courbe peut être décomposée en trois parties : zone de grand bruit au début de la courbe, zone plate et puis une zone de décroissance. La fin de la zone plate correspond à la tension de sortie proportionnelle au nombre des photons enregistrés par le PM. Si le PM est en régime spectrométrique la fonction de réponse est linéaire. Il est donc nécessaire de rechercher avec soin la zone de fonctionnement linéaire pour repérer le plateau. Les trois courbes correspondant aux trois niveaux d'eau ont une forme similaire en échelle semi logarithmique. On peut alors supposer qu'elles étaient la somme de deux fonctions de type (échelle log-log) $Y(x)=a+b x^{c}$ avec les exposants $c$ variables. Une approximation d'une telle fonction a eu pour objectif l'obtention du point de début de la décroissance qui correspondait au courant de sortie en régime linéaire du PM. Pour les trois niveaux de l'eau on a obtenu trois courbes différentes et avec l'approximation initiale on a trouvé les trois points de la tension de sortie. Les deux courbes (la courbe théorique : le nombre des photons Tcherenkov calculé par EGS4, la courbe expérimentale des trois tensions de sortie du PM) sont représentées sur la figure 3d (Mishev et al., 2001a, 2001b; Mishev, 2002). On a constaté une évolution identique de leurs allures. Bien qu'on ait utilisé une petite cuve différente de celles utilisées dans le télescope, les résultats obtenus peuvent être considérés comme une validation directe du modèle. Il est évident que si le modèle est confirmé pour une géométrie, il sera utilisable pour d'autres géométries comparables en tenant compte des limitations d'EGS4.

Après les études expérimentales avancées (Mishev et al., 2001a, 2001b) du modèle, des changements additionnels ont été effectués dans le code. Le remplacement de la masse de l'électron au repos par la masse du muon permet en 

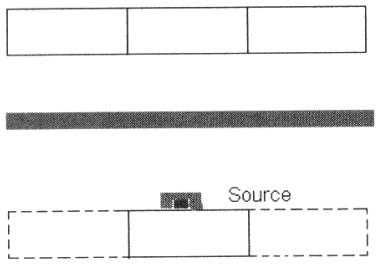

$\mathrm{Pb}$

module inférieur

scintillateur

(a)

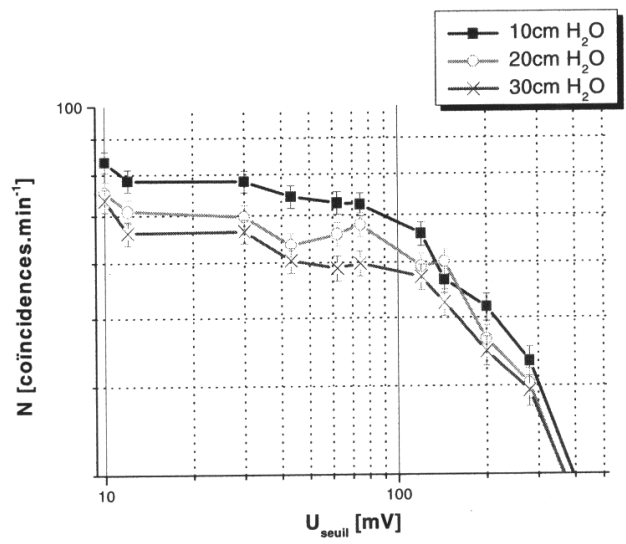

(c)

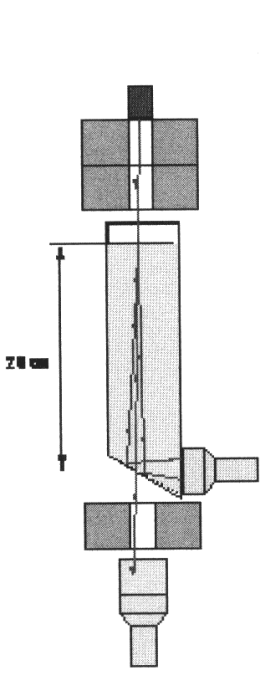

(b)

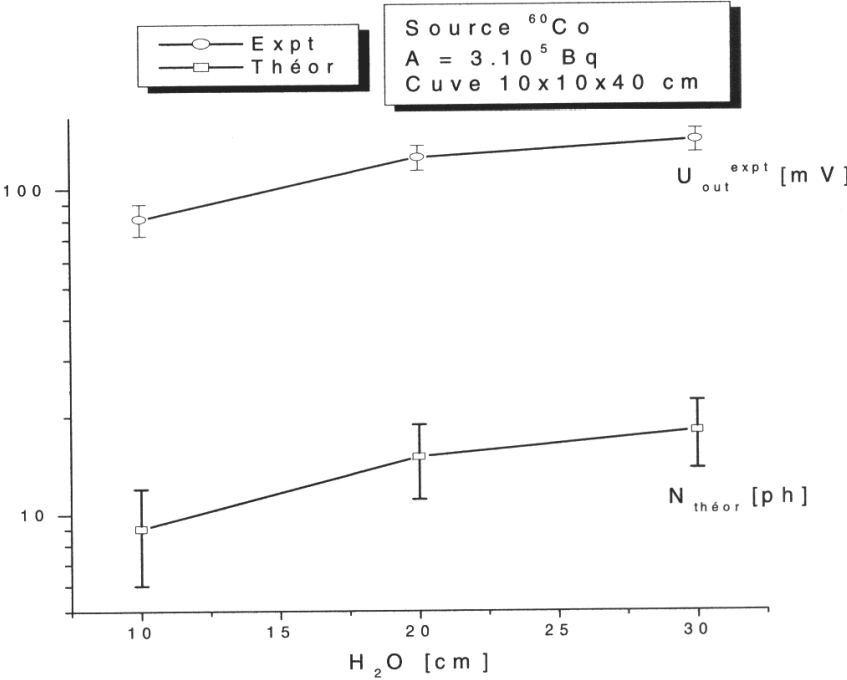

(d)

Figure 3 - (a) Le module supérieur du télescope en anticoüncidence avec le module de mesure. (b) Petite cuve de mesures. (c) Taux de comptage en fonction du seuil du PM pour 10, 20 et $30 \mathrm{~cm}$ de l'eau. (d) Résultats expérimentaux et courbe théorique.

(a) The upper module of the telescope in anticoincidence with the measuring tank. (b) Small water tank. (c) Counting rate for 10,20 and $30 \mathrm{~cm}$ water depth. (d) Experimental and simulated response of a small tank. 


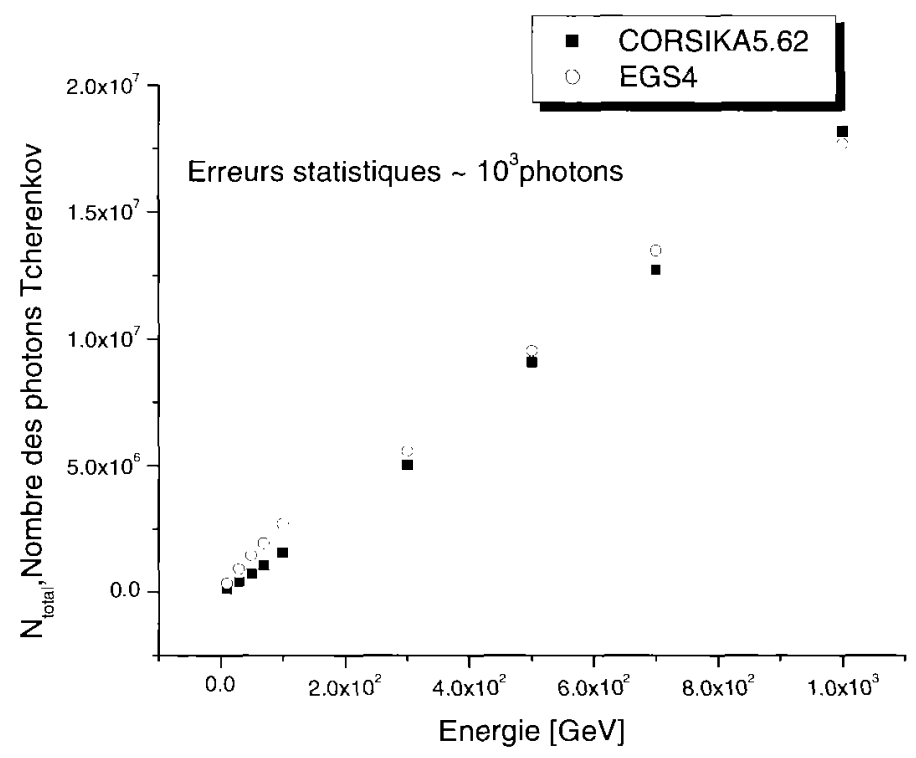

Figure 4 - Comparaison entre les codes EGS4 et CORSIKA (Mishev et al., 2001a, 2001b).

Comparison between EGS4 and CORSIKA codes (Mishev et al., 2001a, 200Ib).

première approximation la simulation de la réponse du télescope muonique. Il semble que ce soit une bonne approche (les pertes d'énergie des muons dans $10 \mathrm{~cm}$ d'eau sont négligeables et la différence entre la quantité calculée avec EGS4 et la quantité «théorique » est $<10 \%$ ). Malgré les différences entre le muon et l'électron la simulation de la production du rayonnement Tcherenkov se montre raisonnable dans les conditions limitées (le cas du télescope muonique $10 \mathrm{~cm}$ ).

Toutes les histoires sont pour des muons incidents distribués uniformément. Le résultat donne une efficacité de $72 \%$ (pour l'énergie du muon de $1 \mathrm{GeV}$ ).

Un modèle simple de l'atmosphère a été introduit dans EGS4 et les simulations pour le rayonnement Tcherenkov produit dans une GGA par des gamma primaires ont été effectuées. L'atmosphère a été divisée en 21 couches de $5 \mathrm{~km}$.

Les sections efficaces pour les différentes couches ont été calculées avec PEGS4 en tenant compte de la pression et de la concentration. Le changement d'indice de réfraction en fonction de l'altitude a été également pris en compte. La comparaison avec le code CORSIKA (logiciel plus complexe utilisant une version modifiée d'EGS4) a été effectuée (Fig. 4) (Mishev et al., 2001a, 200lb). 
Les différences sont dues essentiellement aux différents modèles atmosphériques utilisés et au transport des particules chargées dans CORSIKA. Bien que le modèle utilisé dans EGS4 soit assez simple, il offre la possibilité de développer de nouvelles techniques d'estimation de la transparence de l'atmosphère basées sur l'absorption de la lumière (dans la version CORSIKA 5.62 l'absorption des rayons Tcherenkov n'est pas prise en compte). Il permet également d'utiliser différentes composantes atmosphériques dans des proportions différentes et de poursuivre plus en détail le développement des cascades EM.

Les codes supplémentaires TRAMEAN et TRAJ (Mishev, 2002), traitant les phénomènes optiques dans la cuve d'un détecteur Tcherenkov à base d'eau qui ne sont pas simulés par EGS4, ont été développés. Il s'agit des codes Monte Carlo destinés à l'estimation de la trajectoire des photons Tcherenkov et à l'efficacité d'enregistrement.

En première approche, la masse au repos de l'électron a été remplacée par la masse au repos du muon et l'efficacité est calculée avec l'énergie seuil ( $1 \mathrm{GeV}$ ) du télescope muonique pour la cuve du télescope $(50 \times 50 \times 10 \mathrm{~cm})$ et pour 10000 histoires. Le point d'entrée des muons est choisi aléatoirement entre 0 et $50 \mathrm{~cm}$ pour les coordonnées $X$ et $Y$. L'angle d'inclinaison par rapport à l'axe $Z$ se situe entre 0 et 16 degrés, ce qui correspond au champ actuel du télescope. Avec une énergie de la particule incidente de $1 \mathrm{GeV}$, l'efficacité obtenue a été de $72 \%$. Les mêmes calculs ont été effectués pour une énergie de la particule incidente de $10 \mathrm{GeV}$ et ont donné une efficacité de $68 \%$.

Le code TRAJ se limite à la géométrie cartésienne. L'analogie avec UPHI de EGS4 donne la possibilité de l'introduire directement comme macro et de l'utiliser pour calculer en temps réel la réponse d'un compteur Tcherenkov à base d'eau. L'adaptation à une autre géométrie n'est pas difficile et il est possible à l'avenir de l'utiliser pour résoudre des problèmes similaires.

La structure d'EGS4 permet directement d'intégrer l'effet Tcherenkov comme macro et de l'utiliser pour des calculs rapides. La logique des codes supplémentaires permet également de l'intégrer comme des macro.

\section{Estimation de l'énergie et détermination du type de la particule primaire du rayonnement cosmique basées sur l'enregistrement du rayonnement Tcherenkov atmosphérique}

L'un des principaux problèmes de la physique du rayonnement cosmique est la détermination précise du spectre de rayonnement primaire, surtout dans la région 


\section{A. MISHEV et al.}

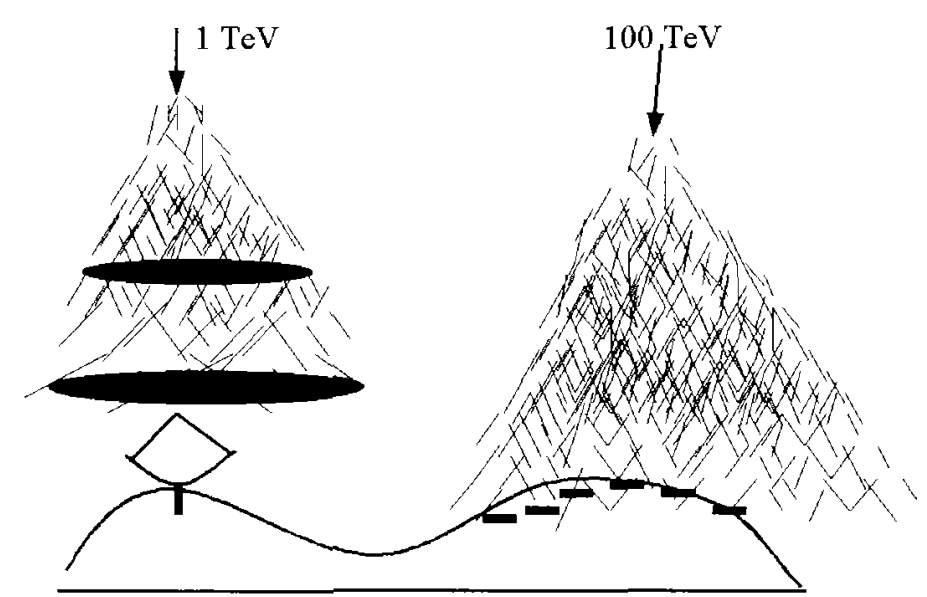

Figure 5 - Principe de détection des photons Tcherenkov dans une GGA créée par des protons primaires de $1 \mathrm{TeV}$ et de $10 \mathrm{TeV}$.

Principe of the Cerenkov photons detection in a GCA created by primary photons between $1 \mathrm{TeV}$ and $10 \mathrm{TeV}$.

des énergies entre $10^{15}$ et $5 \times 10^{15} \mathrm{eV}$. L'amélioration de la statistique des expériences existantes ne peut que partiellement répondre à la question, à laquelle s'ajoute un autre problème important : la composition chimique du rayonnement primaire (surtout la partie haute énergie). Ces études sont relativement difficiles, car les fluctuations rendent presque impossible la reconstruction exacte des caractéristiques des cascades provoquées par différentes particules primaires.

Il existe différentes solutions à ce problème. L'une des possibilités offerte est basée sur l'enregistrement du rayonnement Tcherenkov émis par les particules chargées de la gerbe (Fig. 5), en particulier la détermination des densités des photons Tcherenkov exprimées en photons par $\mathrm{m}^{2}$ et par la suite sur la solution d'un problème inverse non linéaire (Alexandrov et al., 1998, 1999).

L'idée initiale était d'élaborer, suite aux traitements des résultats expérimentaux déjà disponibles, une description de la distribution des densités des photons Tcherenkov dans les GGA et par la suite de chercher une approximation de la distribution latérale du rayonnement Tcherenkov (Alexandrov et al., 1998).

Dans le cas général, la fonction $Q=Q\left(E, R, H, \theta, \varphi, \alpha, H_{0}\right)$ de la distribution latérale du rayonnement Tcherenkov (FDLRT) dépend du type et de l'énergie de la particule incidente, de la distance à l'axe de la gerbe, du niveau d'observation, des coordonnées de la gerbe au niveau de l'observation, de la hauteur de la 
première interaction etc. ( $E$ est l'énergie de la particule primaire, $R$ la distance de l'axe de la gerbe, $H$ le niveau de l'observation, $H_{0}$ la hauteur de la première interaction de la particule primaire - le début de la cascade, $\alpha$ le type de la particule, $\theta$ et $\varphi$ les coordonnées angulaires de la gerbe).

Si le niveau d'observation est fixé, dans le cas d'une expérience donnée, la fonction pour une particule primaire donnée devient $Q=Q(R, E, \theta, \varphi)$ et on peut alors considérer dans ce cas le niveau de la première interaction comme une partie des fluctuations physiques. Si l'angle zénithal est $\theta=0^{\circ}$ pour une énergie donnée, la fonction se simplifie à $Q=Q(R ; p)$ où $p$ sont des paramètres fonction de l'énergie et du type de la particule primaire, $R$ la distance de l'axe de la gerbe. La dépendance de l'angle azimutal n'est pas prise en compte, car dans le cas de $\theta=0^{\circ}$ il existe une symétrie rotationnelle. On peut donc chercher un modèle d'approximation de la FDLRT pour une particule et pour une énergie donnée. Par la suite, la même procédure a été utilisée dans une bande énergétique et pour d'autres particules (Mishev, 2002).

Ainsi, la possibilité d'obtenir la FDLRT peut être basée sur des données simulées. Le code CORSIKA (Knapp et al., 1996) avec les sous programmes VENUS et GHEISHA (Fessenfeldt, 1985) a été utilisé pour la simulation des 500 histoires de GGA par énergie dans une large bande énergétique $\left(10^{13}-10^{16} \mathrm{eV}\right)$. Tous les photons Tcherenkov sont enregistrés par un détecteur de $800 \times 800 \mathrm{~m}$, nécessaire à la détermination correcte de la FDLRT.

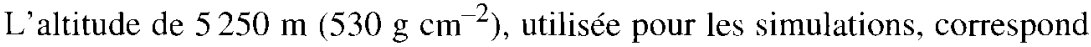
au niveau de la station cosmique de Chacaltaya en Bolivie. Les particules primaires étudiées furent le proton, l'hélium, et le fer. Pour les besoins du modèle, des simulations identiques ont été effectuées pour des gamma primaires (Alexandrov et al., 1998). La bande énergétique était comprise entre $10 \mathrm{TeV}$ et $10 \mathrm{PeV}$ pour le noyaux et entre $1 \mathrm{TeV}$ et $1 \mathrm{PeV}$ pour les gamma.

Les résultats des calculs présentés sur la figure 6a (proton primaire à titre d'exemple) ont été suivis par la recherche d'une fonction d'approximation de ces distributions. Bien que la quantité des photons Tcherenkov au niveau de l'observation de Chacaltaya représente environ $75 \%$ de la totalité de la gerbe, le développement de la cascade est proche de son maximum. Par conséquent, les fluctuations des fonctions de la distribution latérale se montrent moins importantes. Cela facilite d'un côté l'approximation et de l'autre côté cela permet de trouver des critères pour déterminer les caractéristiques de la particule incidente. La fonction recherchée doit être une bonne description de la FDLRT, monotone, avec un gradient diminuant en fonction de la distance $R$ et enfin elle doit être intégrable (pour les besoins de la méthodologie proposée). 


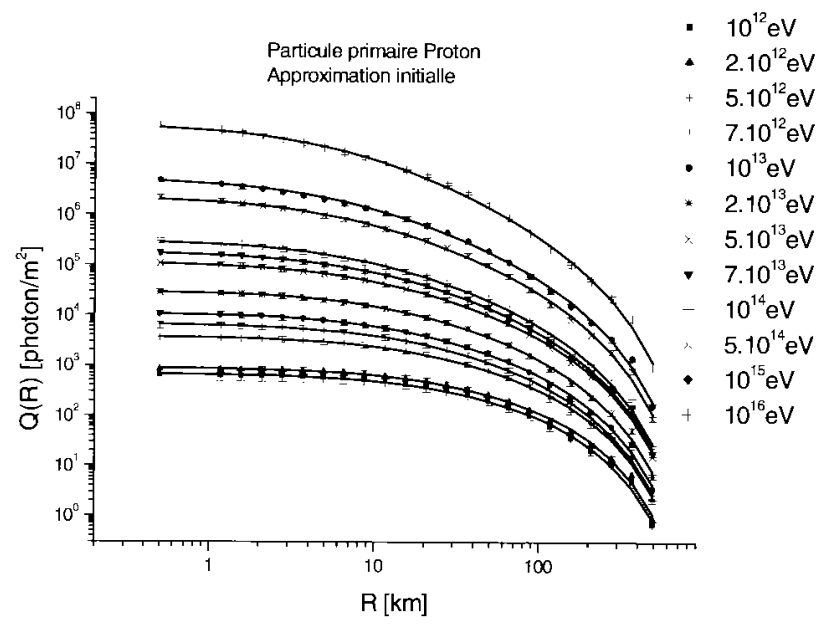

(a)

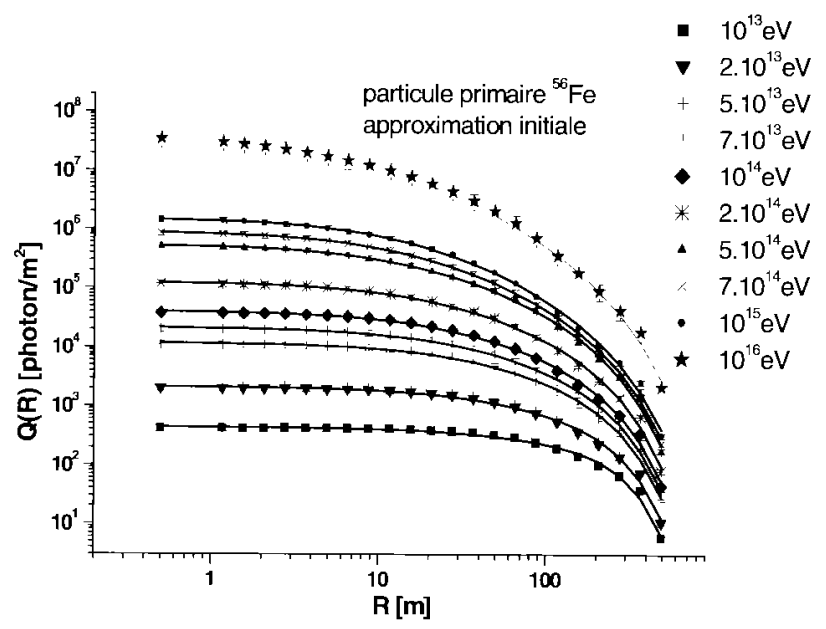

(b)

Figure 6 - (a) Fonction de la distribution latérale du rayonnement Tcherenkov pour protons. Données simulées avec le code CORSIKA 5.62 (GHEISHA et VENUS sous programmes), altitude d'observation $530 \mathrm{~g} \mathrm{~cm}^{-2}, 500$ histoires par énergie. (b) Fonction de la distribution latérale du rayonnement Tcherenkov pour fer. Données simulées avec le code CORSIKA 5.62 (GHEISHA et VENUS sous programmes), altitude d'observation $530 \mathrm{~g} \mathrm{~cm}^{-2}, 500$ histoires par énergie.

(a) Function of the lateral distribution of Cerenkov ray for protons. Simulated data with CORSIKA 5.62 (GHEISHA and VENUS program), altitude of observation $530 \mathrm{~g} \mathrm{~cm}^{-2}$, 500 histories by energy. (b) Function of the lateral distribution of Cerenkov ray for iron. Simulated data with CORSIKA 5.62 (GHEISHA and VENUS program), altitude of observation $530 \mathrm{~g} \mathrm{~cm}^{-2}, 500$ histories by energy. 


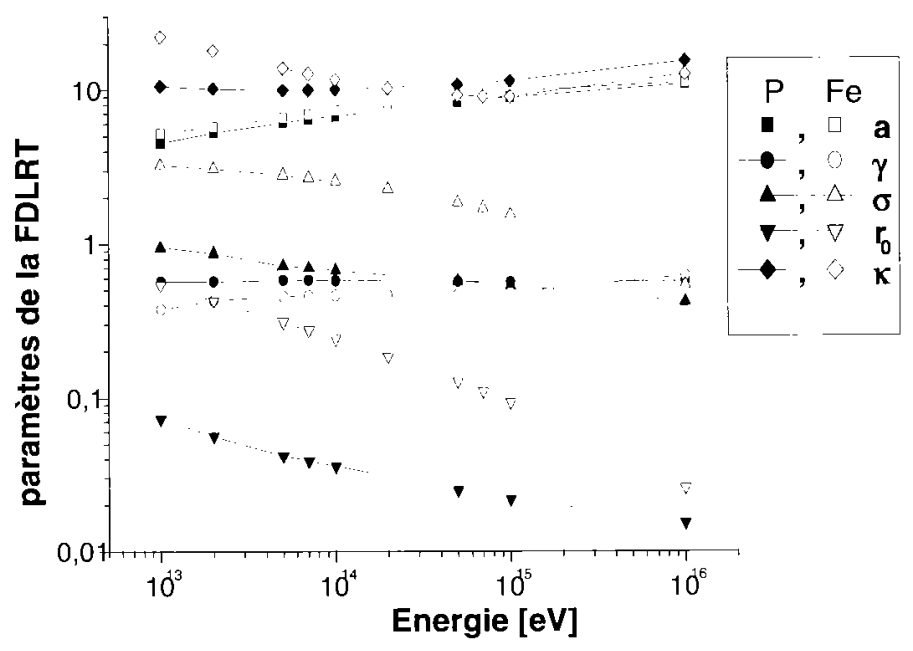

Figure 7 - Différence entre les paramètres du modèle pour le proton et le fer.

Difference between the model parameters for proton and iron.

Le comportement des paramètres en fonction de l'énergie doit être également monotone. L'algorithme utilisé est basé sur la solution d'un problème inverse en utilisant un processus de Gauss-Newton avec auto régularisation. Cette méthode est réalisée dans le code REGN (Alexandrov, 1971). L'expression trouvée est, en dehors du $R$, la fonction de plusieurs paramètres

$$
Q(R)=\frac{\sigma \mathrm{e}^{a} \mathrm{e}^{-\left[\frac{R}{\gamma}+\frac{R-r_{0}}{\gamma}+\left(\frac{R}{\gamma}\right)^{2}+\left(\frac{R-r_{0}}{\gamma}\right)^{2}\right]}}{\gamma\left[\left(\frac{R}{\gamma}\right)^{2}+\left(\frac{R-r_{0}}{\gamma}\right)^{2}+\frac{R \sigma^{2}}{\gamma}\right]}
$$

avec $a$ l'amplitude, $\gamma$ le premier pôle responsable de la structure près de l'axe de la gerbe, $r_{0}$ le deuxième pôle responsable de la structure loin de l'axe de la gerbe, $\sigma$ la largeur de la distribution et $\kappa$ la jonction avec l'énergie $E=\kappa f\left(N_{q}\right)$ où $N_{q}=$ $2 \pi \cos (\theta)[Q(R) R \mathrm{~d} R$ (Mishev, 2002 ; Alexandrov et al., 2001). Des calculs analogues ont été effectués pour le fer primaire (Fig. 6b). Les paramètres varient en fonction de l'énergie et caractérisent les différentes particules primaires (Fig. 7). Leur approximation s'effectue à l'aide de polynômes qui sont fonction du logarithme de l'énergie. La même procédure a été appliquée par la suite en remplaçant dans le modèle trouvé les paramètres par leurs approximations. Il s'agit 


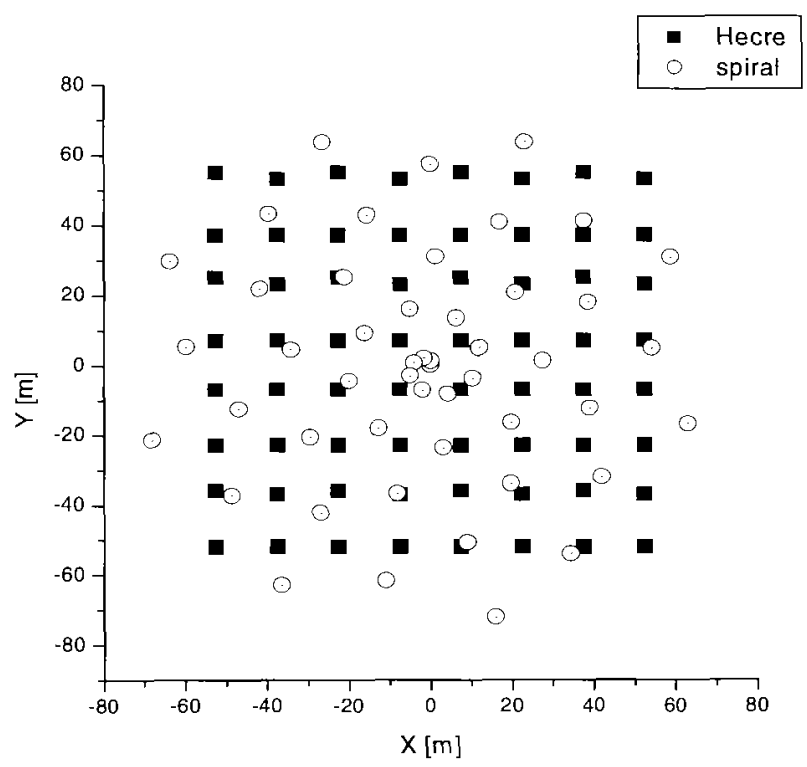

Figure 8 - Configurations de HECRE et SPIRAL.

Configuration of HECRE and SPIRAL.

finalement de la solution d'un problème inverse de deux variables : $R$, la distance de l'axe de la gerbe et $E$, l'énergie de la particule incidente, grâce à laquelle le nombre total des photons Tcherenkov dans la gerbe au niveau de l'observation peut être déterminé. La grande non linéarité du modèle ainsi que la jonction avec l'énergie permet de distinguer le proton du fer primaire et d'estimer l'énergie de la particule primaire.

La méthode trouvée permet donc de réaliser une simulation de la réponse d'un détecteur basé sur l'enregistrement du rayonnement Tcherenkov atmosphérique (expérience de Chacaltaya). L'énergie de la particule primaire prise aléatoirement dans le spectre du rayonnement cosmique, son angle zénithal, son angle azimutal et le point du pied de la gerbe peuvent être ainsi simulés. Le nombre des photons Tcherenkov est calculé pour différentes configurations des détecteurs. La surface du détecteur est également prise en compte. La réponse suit la loi de Poisson si le nombre des photons incidents sur le détecteur est inférieur à 10 , et celle de la distribution de Gauss si le nombre est supérieur à 10. Le nombre des photons Tcherenkov incidents sur les détecteurs est calculé selon deux configurations : HECRE (Saavedra et Jones, 2001) et SPIRAL (Alexandrov et al., 2001) (Fig. 8). 


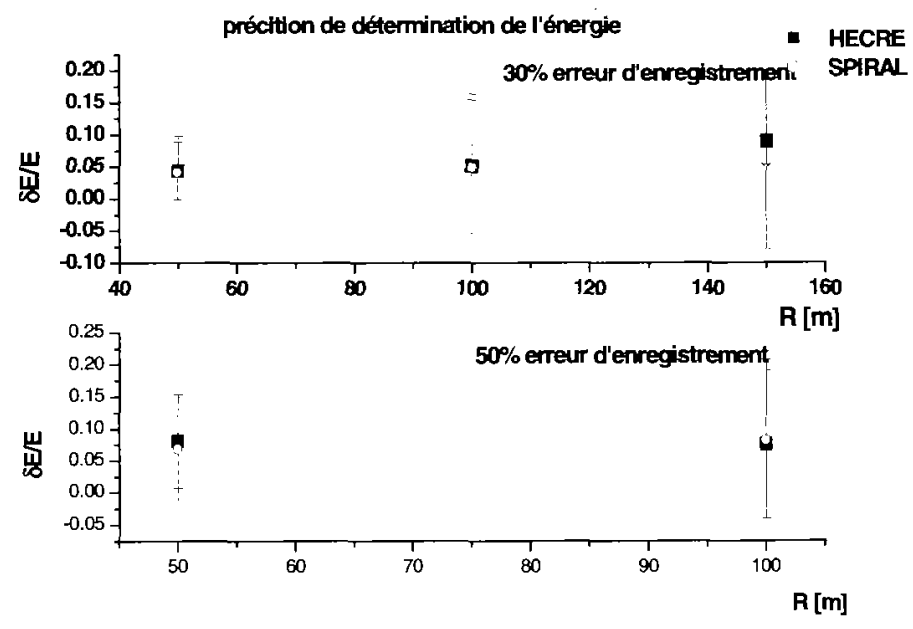

Figure 9 - Précisions de détermination de l'énergie en fonction de la distance du centre de la configuration. Accuracy in energy estimation function of the configuration center.

Il s'agit à nouveau de la solution d'un problème inverse avec la fonction obtenue, mais cette fois à partir de données simulées des détecteurs. Pour les différents pieds de gerbe (angles et énergies), les données simulées et les données obtenues ont pu être comparées (Alexandrov et al., 2001). Les précisions de la détermination de l'énergie sont représentées sur la figure 9 . On constate que dans le cas de « $50 \%$ » erreur d'enregistrement SPIRAL donne une plus grande précision dans le cas où les axes sont à plus de $150 \mathrm{~m}$ du centre de la configuration. Dans le cas de « $30 \%$ » erreur d'enregistrement les deux configurations donnent des résultats similaires.

\section{Conclusion}

Les recherches effectuées dans le cadre de ce travail ont contribué à l'utilisation de différents codes de Monte Carlo pour simuler des cascades électromagnétiques et hadroniques dans divers milieux (EGS4 et CORSIKA 5.62). Les modélisations de l'effet Tcherenkov dans l'eau (EGS4) et dans l'atmosphère (EGS4 et CORSIKA 5.62) ont été effectuées. La mise en place d'un outil permettant l'optimisation future du fonctionnement et l'amélioration des performances du télescope muonique ont été entre autre réalisées. 
Les principaux résultats obtenus peuvent être globalement résumés en quelques points :

- l'effet Tcherenkov a été introduit dans la partie « utilisateur » du code EGS4 et la simulation de la réponse d'un détecteur Tcherenkov à base d'eau en réflexion totale a été effectuée pour différentes géométries de la cuve dans une large bande énergétique ;

- une vérification expérimentale directe de ce simple modèle a été réalisée. Les expériences ont montré la possibilité de simuler correctement la réponse des détecteurs Tcherenkov à base d'eau avec le code EGS4 ;

- des codes additionnels ont été développés pour simuler la trajectoire des photons Tcherenkov dans la cuve. Cela a permis d'estimer l'efficacité d'enregistrement du télescope, qui a été établie à $72 \%$;

- l'utilisation du code CORSIKA, dans la bande d'énergie $10 \mathrm{TeV}-10 \mathrm{PeV}$ au niveau d'observation de Chacaltaya $\left(536 \mathrm{~g} \mathrm{~cm}^{-2}\right.$ ) en Bolivie, donne les fonctions de la distribution latérale des électrons, des muons, des hadrons et des photons Tcherenkov;

- un modèle d'approximation de la fonction de distribution latérale des rayons Tcherenkov a été trouvé grâce à l'utilisation de la solution d'un problème inverse non linéaire ;

- une nouvelle méthodologie pour estimer l'énergie de la particule primaire du rayonnement cosmique et pour déterminer son type (proton et fer), basée uniquement sur l'exploitation de l'effet Tcherenkov, a été proposée.

Dans l'avenir, l'ensemble de cette étude pourra être étendue au développement de systèmes autres que le télescope muonique et servira de base aux projets futurs dans le domaine, particulièrement attractif et intéressant, de la détection du rayonnement cosmique.

Le développement des techniques de détection et la bonne connaissance des champs de rayonnement cosmique représentent également une base de données importante pour une radioprotection efficace et préventive non seulement de l'homme mais aussi pour la protection du matériel de plus en plus souvent exposé et utilisé dans les conditions spécifiques (vols en altitudes, matériel à bord de satellites...). Leur exploitation pour les mesures dans l'environnement (pollutions atmosphériques) peut être également envisagée.

Remerciements. Nous tenons à remercier l'AEN-OCDE, les auteurs des codes (EGS4, CORSIKA) et plus particulièrement D. Heck.

\section{RÉFÉRENCES}

Alexandrov L. (1971) Regularized Newton-Kantorovich Computational Processes, J. Comp. Math. Math. Phys. 11(1), 36-43. 


\section{CONTRIBUTION AU DÉVELOPPEMENT DE NOUVELLES TECHNIQUES}

Alexandrov L., Brankova M., Kirov I., Mavrodiev S., Mishev A., Stamenov J., Ushev S. (1998) Report of the JINR Dubna E2-98-48, Dubna-Russia.

Alexandrov L., Brankova M., Kirov I., Mavrodiev S., Mishev A., Stamenov J., Ushev S. (1999) Report of the JINR Dubna E2-99-233, Dubna-Russia.

Alexandrov L., Mavrodiev S., Mishev A., Stamenov J. (2001) Estimation of the Primary Cosmic Radiation Characteristics, Proc. of XXVII ICRC Hamburg, pp. 257-261.

Angelov I., Kalapov I., Malamova E., Stamenov J. (1999) Muon Chercnkov Telescope, 8th National Scientific and Applied Conference, 22-24 September, Sozopol (Bulgaria).

Duverger E., Makovicka L., Mishev A., Stamenov J. (2000) Modelling of Ccrcnkov effect with CORSIKA and EGS4 codes, Internat. Conf. on Advanced Monte Carlo for Rad. Physics, Particle Transport Simulation and Applications, 23-26 Oct. 2000, Lisbon.

Fessenfeldt H. (1985) Report PITHA-85/02, RWTH Aachen.

Heck D., Knapp J., Capdevielle J.N., Schatz G., Thouw T. (1998) CORSIKA a Monte Carlo code to simulate extensive air showers, Report FZKA 6019, Forschungszentrum Karlsruhe, KarlsruheGermany.

Knapp J., Heck D., Schatz G. (1996) Report FZKA5828, Forschungzentrum Karlsruhe, Germany.

Malamova E., Angelov I., Kalapov I., Davidkov K., Stamenov J. (2001) Muon Cherenkov Telescope, Proc. of 27th ICRC, 07-15 August 2001, Hamburg, pp. 3952-3955.

Mishev A., Duverger E., Makovicka L., Stamenov J. (2001a) Modelling of the Ccrenkov effect, Radiat. Phys. Chem. 61, 371-373.

Mishev A., Angelov I., Duverger E., Gschwind R., Makovicka L., Stamenov J. (2001b) Experimental study and Monte Carlo modeling of the Cerenkov effect, Nucl. Instr. Meth. Phys. Res. A 474, $101-107$.

Mishev A. (2002) Études théoriques et expérimentales de l'effet Tcherenkov dans différents milieux pour une large bande énergétique. Application en détéction des rayonnements cosmiques, thèse № 68/UFR STGI, université de Franche-Comté.

Nelson W., Hirayama H., Rogers D.W.O. (1985) The EGS4 code system, SLAC Report 265, Stanford, CA 94305.

Saavedra O., Jones L. (2001) Chacaltaya: towards a solution of the knee, Nuovo Cim. C 24, 497-506. 